\title{
Isolation and Toxigenic Characteristics of a Tetrodotoxin Producing Bacteria
}

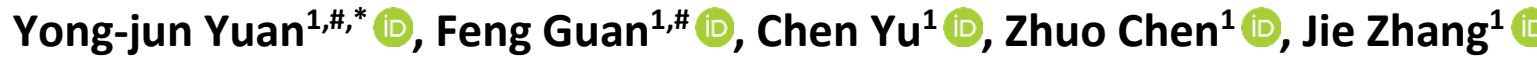 \\ ${ }^{1}$ Zhejiang Wanli University, College of Biological \& Environmental Sciences, Ningbo/ Zhejiang, China (315100). \\ \#These authors share the first authorship.
}

\section{How to cite}

Yuan, Y., Guan, F., Yu, Y., Chen, Z., Zhang, J. (2022). Isolation and Toxigenic Characteristics of a Tetrodotoxin Producing Bacteria. Turkish Journal of Fisheries and Aquatic Sciences, 22(1), TRJFAS19531. http://doi.org/10.4194/TRJFAS19531

\section{Article History}

Received 25 March 2021

Accepted 25 August 2021

First Online 28 August 2021

Corresponding Author

Tel.: +8657488222336

E-mail: yuan2007019@163.com

\section{Keywords}

Tetrodotoxin

Inducer

Bacillus cereus

Identification

\begin{abstract}
Tetrodotoxin (TTX) is a kind of neurotoxin with great scientific research and medicinal value. Studies have confirmed that microorganism is one of the important sources of TTX. However, the specific biosynthetic and regulatory pathway of TTX production by microorganisms are still unclear. In this paper, out of twelve symbiotic bacteria screened from the wild puffer fish (Fugu rubripes) collected from coastal sea area of Ningbo (Zhejiang, China), a strain named He-1 isolated from the liver of puffer fish was found can produce TTX. It was identified as Bacillus cereus based on physiological, biochemical characteristics and 16S rDNA gene analysis. However, the strain He-2, which is the subculture strain of He-1, cannot produce TTX under the normal culture condition. Further study showed that by adding the metabolite of the He-1, the ability of TTX producing of He-2 could be restored. A peptide compound was obtained by separating and purifying of the metabolites from strain $\mathrm{He}-1$, which could induce the TTX production of strain He-2. This work will provide a new way for further revealing the anabolic pathway of TTX.
\end{abstract}

\section{Introduction}

Despite its well-known toxicity, there is a very long history of pufferfish (Fugu rubripes) consumption in Asian countries such as China and Japan. Early studies shown that tetrodotoxin (TTX; $\mathrm{C}_{11} \mathrm{H}_{17} \mathrm{~N}_{3} \mathrm{O}_{8}$ ) is the cause of lethality after pufferfish consumption (Hwang \& Noguchi, 2007; Rocher et al., 2011; Pratheepa \& Vasconcelos, 2013). Further studies have shown that TTX widely exists in many organisms, such as shellfish, mollusks, arthropods, reptiles, and amphibians (Noguch \& Arakawa, 2008; Bane et al., 2014; Turner et al., 2015). In addition to being a cause of acute food poisoning, chronic accumulation of TTX in the human body may be responsible for amyotrophic lateral sclerosis (ALS) (Sanford et al., 2005).

TTX is a neurotoxin with marked scientific and medicinal value. It is a highly effective $\mathrm{Na}^{+}$channel blocker that is an irreplaceable tool in neurobiology and physiology research (Steffens et al., 2003). TTX has numerous bioactivities, including blood pressurelowering, anti-arrhythmia, spasm-relieving, analgesic, and local anesthetic effects, and it has been previously used clinically (Padera et al., 2006; Nieto et al., 2012). When used for detoxification and cancer pain suppression, TTX has several advantages, as only a low 
dose is required, and it is long-acting and non-addictive (Hagen et al., 2008; Song et al., 2011).

Conventionally, TTX is either produced through chemical synthesis or purified from organisms. However, chemical synthesis is an expensive process that involves complex, time-consuming reactions and results in low yields (Nishikawa \& Isobe, 2013). Conversely, the purification of TTX from the ovaries and livers of wild pufferfish is a relatively inefficient process and has a detrimental impact on aquatic life (Kudo et al., 2014). Therefore, a more reliable, cost-effective, and sustainable method of producing TTX is needed.

There is consensus regarding the source of TTX (Chau et al., 2011), and many studies have confirmed that microorganisms could be essential sources for the production of TTX (Pratheepa \& Vasconcelos, 2013). As an increasing number of TTX-producing microorganisms are isolated and identified, researchers have begun to study the feasibility of large-scale TTX production via microbial fermentation. Research and development in this area includes screening for TTX-producing strains, optimizing fermentation conditions, and mutagenesisbased breeding of wild TTX-producing strains (Campbell et al., 2009; Tu et al., 2014). Although some progress has been made in the research on TTX production via microbial fermentation, a central problem remains, which is the instability of microbial TTX production. Studies have shown that the wild strains have high TTX production ability in their symbiotic partners; however, this ability typically decreases sharply after isolation. Wild strains that show high TTX production ability in early generations often exhibit decreased or complete loss of TTX production ability after several subcultures, making follow-up research and subsequent applications difficult (Yu et al., 2011; Magarlamov et al., 2014). Therefore, more research needs to be performed on the biosynthetic pathway and mechanisms that regulate TTX production by microorganisms.

In the present study, a TTX-producing strain from a wild pufferfish was isolated and identified. The regulation of TTX production in a subcultured nonproducing strain was explored by adding metabolites from the original TTX-producing strain. A metabolite that could induce TTX production was isolated and identified. Our study aims to provide a new method to analyze the anabolic pathway employed by microorganisms in TTX production.

\section{Materials and Methods}

\section{Reagents and culture media}

TTX standard and all other chemicals were purchased from Aladdin (Shanghai Aladdin Biochemical Technology Co., Ltd.). Sterilized seawater medium was used for culturing bacteria. The medium contained (w/v) $2 \mathrm{~g} / \mathrm{L}$ peptone, $2 \mathrm{~g} / \mathrm{L}$ yeast extract, and $1 \mathrm{~g} / \mathrm{L}$ glucose, and it was prepared by adding seawater (up to $1 \mathrm{~L}$ ) from the area where the pufferfish were caught. Agar plates were prepared by adding $15 \mathrm{~g}$ of agar to $1 \mathrm{~L}$ of seawater medium.

\section{Collection and pretreatment of samples}

All animal experiments were carried out according to the animal ethics guidelines of Zhejiang Wanli University. In March 2018, an adult wild pufferfish was caught from the sea of the coast of Ningbo and transported alive to the laboratory at Zhejiang Wanli University. Once in the laboratory, different tissues were aseptically excised from the specimen for immediate isolation of bacteria.

\section{Screening of TTX-producing bacteria}

The liver, ovaries, and intestines were homogenized in a sterile blender, transferred to a conical flask containing $100 \mathrm{~mL}$ of sterile seawater, and incubated with shaking at $180 \mathrm{rpm}$ for $20 \mathrm{~min}$. Bacteria were screened by spreading $1 \mathrm{~mL}$ of a diluted sample on an agar plate and then incubating the plate at $25{ }^{\circ} \mathrm{C}$ for $5 \mathrm{~d}$. Typically, a mixture of colonies formed on each plate that were selected and purified using the streak-plate method on a new agar plate until discrete colonies were obtained. Based on their color and morphology, 12 different colonies were selected, and their TTX production capacity was determined using an enzymelinked immunosorbent assay (ELISA) for TTX (Wuhan Unibiotest Co., Ltd., China).

\section{Identification of a TTX-producing strain}

Strain He-1 was characterized according to standard procedures described in the Manual of Methods for General Bacteriology (Gerhardt, 1981). For performing phylogenetic analysis, genomic DNA was extracted, the $16 \mathrm{~S}$ rDNA gene was amplified by PCR, and the purified PCR products were sequenced. The $16 \mathrm{~S}$ rDNA gene sequence of $\mathrm{He}-1$ was compared with sequences in the GenBank database using BLAST. Multiple sequence alignments were generated with the ClustalX program, and a phylogenetic analysis was conducted with the MEGA program.

\section{Identification of TTX}

TTX was purified from the cultured bacteria as previously described by Maren et al. (2007). TTX purified from a culture of strain He-1 was identified by thin-layer chromatography (TLC). The test samples and TTX standard were separated on precoated silica gel plates (Qingdao Marine Chemical Corporation, China). The solvent system used was pyridine: ethyl acetate: glacial acetic acid: water (8:5:3:3, v/v). Both the TTX standard and test samples were applied to the TLC plate using capillary tubes. After the plate was sprayed with $10 \%$ $\mathrm{KOH}$ and heated at $110^{\circ} \mathrm{C}$ for $10 \mathrm{~min}$, TTX was visualized 
as green or blue fluorescent spots under UV light (365 $\mathrm{nm})$.

\section{Quantification of TTX}

To quantify the TTX content in a sample, a TTX ELISA test kit was used, which utilizes a monoclonal antibody against TTX and the competitive ELISA method. According to the procedure described in the manual, the Log of the ratio of the absorbance of the sample to the absorbance of the blank without $\operatorname{TTX}\left(A_{s} / A_{0}\right)$ has a linear relationship with the logarithmic concentration of TTX in the sample within a specific concentration range. Therefore, the TTX concentration in the sample was calculated based on a TTX standard curve.

\section{Induction of TTX production}

Strain $\mathrm{He}-2$ is the second generation of strain $\mathrm{He}-1$, which was obtained by subculture in standard culture medium (sterilized seawater medium) that cannot produce TTX. To determine whether TTX production could be induced in He-2 using medium from the TTXproducing strain, $\mathrm{He}-1$, medium from a culture of strain He-1 was purified as described below. After each purification step, the purified sample was added to the culture medium of strain $\mathrm{He}-2$. After cultivation for 5-7 $\mathrm{d}$, TTX content in the culture broth of strain He-2 was assayed using the TTX test kit.

Isolation and purification of an inductive substance
The culture broth of strain He-1 was filter-sterilized and concentrated. Then, as the first purification step, ultrafiltration was performed using membranes with different molecular weight cutoffs (MWCO; 1,3 , and 5 $\mathrm{kDa}$ ). Components of different molecular weights ( $\leq 1$ $\mathrm{kDa}, 1-3 \mathrm{kDa}$, and $\geq 3 \mathrm{kDa}$ ) were collected, condensed by vacuum rotation, and finally freeze-dried. The induction activity of the separated products with different molecular weights was detected as described above.

Next, gel chromatography was performed on a Sephadex G-10 column $(2.6 \mathrm{~cm} \times 60 \mathrm{~cm})$. Samples were eluted with distilled water at a rate of $0.3 \mathrm{~mL} / \mathrm{min}$, collected using an automatic fraction collector with monitoring at $280 \mathrm{~nm}$, and lyophilized. The induction activity of each fraction was detected as described above.

After gel chromatography, a Shimadzu high performance liquid chromatography (HPLC) system (Shimadzu Corporation, Kyoto, Japan) was used to further separate and analyze the samples. The separation was conducted using mobile phases $A(0.1 \%$ TFA in water) and $B(0.1 \%$ TFA in acetonitrile) at a flow rate of $1 \mathrm{~mL} / \mathrm{min}$. The injection volume was $25 \mu \mathrm{L}$, and the absorbance was monitored at $220 \mathrm{~nm}$. Fractions were collected and lyophilized, following which their induction activity was analyzed.

\section{Analysis of the amino acid composition of the purified peptide}

RP-HPLC-MS/MS was used to determine the sequence of the peptide in the fraction. Mobile phase $A$ was $100 \%$ acetonitrile and mobile phase $B$ was water

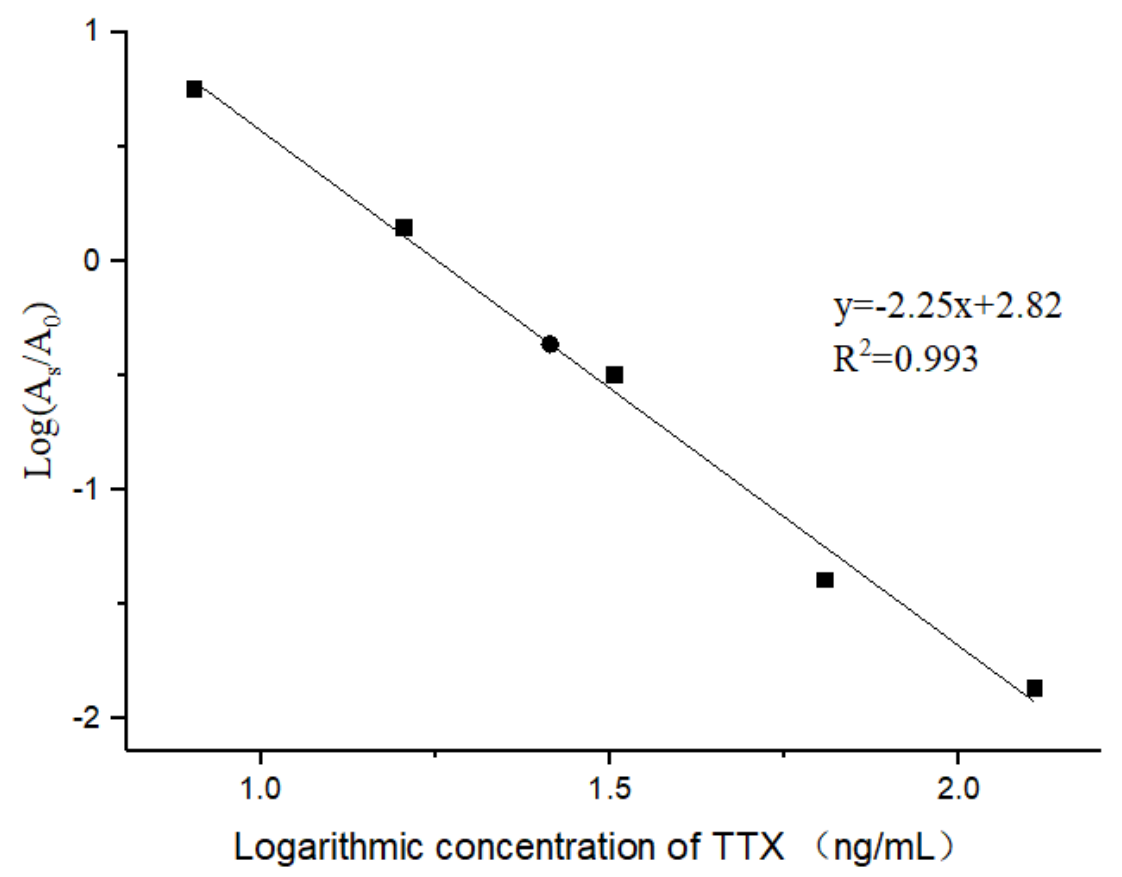

Figure 1. Standard curve established with the TTX ELISA test kit. Range of TTX concentration: 10-100 ng/mL. 
with $0.1 \%(\mathrm{~m} / \mathrm{v})$ trifluoroacetic acid, and the separation was carried out by gradient elution ( $5 \% \mathrm{~A}$ to $50 \% \mathrm{~A}$ at a flow rate of $0.5 \mathrm{~mL} / \mathrm{min}$ in $40 \mathrm{~min}$ ). The conditions for mass spectrometry were as follows: electrospray ionization; atomizing gas pressure, 20 psi; dry gas $\left(\mathrm{N}_{2}\right)$ flow rate, $0.8 \mathrm{~L} / \mathrm{min}$; dry temperature, $150{ }^{\circ} \mathrm{C}$; spectral range [mass/charge $(\mathrm{m} / \mathrm{z})$ ], 50 to 3000 ; and capillary voltage, $3300 \mathrm{~V}$.

\section{Result}

\section{Screening of TTX-producing strains}

In total, 12 strains were isolated from the pufferfish, and only one of them produced TTX. A standard curve was established using the TTX ELISA test kit (Figure 1). This strain was named He-1 and subjected to molecular identification.

\section{Confirmation of the identity of TTX in the culture medium of strain He-1}

TTX can be hydrolyzed by alkali to form $C_{9}$ base (2amino-6-hydroxymethyl-8-hydroxyquinazoline), which fluoresces under UV light. In the TLC analysis, a fluorescent spot purified from the culture of $\mathrm{He}-1$ was visualized that had the same $R_{f}$ value as standard TTX $\left(R_{f}\right.$ $=0.58$ ). Other fluorescent spots observed in the sample were not found in the TTX standard, and thus, they may be TTX analogs or other compounds (Figure 2).

\section{Identification of strain He-1}

A preliminary morphological and biochemical survey showed that strain $\mathrm{He}-1$ might belong to the genus Bacillus. Hence, detailed experiments to determine the physiological and biochemical characteristics were carried out, and the results are summarized in Table 1. As shown, the strain that was closest to He-1 was Bacillus cereus ATCC 11778.

The molecular identification of strain $\mathrm{He}-1$ was based on the 16S rDNA gene sequence analysis. The $16 \mathrm{~S}$ rDNA sequence of strain He-1 was compared with the sequences of other bacterial species in GenBank, and the newly isolated strain was identified as Bacillus cereus, as it shared the highest similarity (99\%) with this species. Therefore, strain He-1 was named Bacillus cereus He-1 and deposited in GenBank under accession number MN826691.

An annular phylogenetic tree (Figure 3) was generated using $16 \mathrm{~S}$ rDNA partial sequences from 10 Bacillus cereus strains using the neighbor-joining method, which showed eight clusters. Based on this phylogenetic analysis, strain $\mathrm{He}-1$ was identified as Bacillus cereus.

\section{Toxicity of different He-1 subcultures}

Strain He-1 was subcultured for several generations, and the TTX production capacity of each generation was determined using the TTX ELISA test kit. The results showed that TTX could not be detected in the second generation of subculture (strain $\mathrm{He}-2$ ).

\section{Induction of TTX production by the subcultured strain}

After filter-sterilization and quantification of TTX, the culture broth of the primary strain, He-1, was added to the medium used to grow strain He-2. After culture, TTX was detected in the culture broth of strain $\mathrm{He}-2$, and the TTX content in the $\mathrm{He}-2$ culture broth was significantly higher than that added from the culture broth of He-1. This indicated that strain He-2 produced TTX during culture and that there was some substance in the culture broth of $\mathrm{He}-1$ that induced strain $\mathrm{He}-2$ to produce TTX.

\section{Isolation and identification of the inducer}

After ultrafiltration, samples of culture broth from strain He-1 were separated into three fractions, named $\mathrm{U} 1, \mathrm{U} 2$, and $\mathrm{U} 3$, representing the ultrafiltration products with molecular weights $\leq 1 \mathrm{kDa}, 1-3 \mathrm{kDa}$, and $\geq 3 \mathrm{kDa}$, respectively. Induction activity was determined after adding these three ultrafiltered samples, and the results showed that only U2 had TTX production-inducing activity.

The U2 sample was further separated by gel chromatography and divided into four fractions named

Table 1. Physiological and biochemical characteristics of the strain He-1

\begin{tabular}{lcc}
\hline Items & Bacillus cereus ATCC 11778 & Bacillus cereus He-1 \\
\hline Nitrate broth & No change color & No change color \\
Amylolysis & No change color & No change color \\
Simons citrate & Green & Green \\
VP test & Reddish & Reddish \\
Glucose & Yellow & Yellow \\
Mannitol & Green & Green \\
Lysozyme broth & Turbid & Turbid \\
Motility medium & Diffusion growth & Diffusion growth \\
Gelatin medium & Liquid at $4{ }^{\circ} \mathrm{C}$ & - \\
Catalase reagent & No bubbles & + \\
\hline
\end{tabular}


G1, G2, G3, and G4, respectively (Figure 4). The second fraction, $\mathrm{G} 2$, showed induction activity.

Subsample G2 was further separated by HPLC, and four peaks, named $\mathrm{H} 1, \mathrm{H} 2, \mathrm{H} 3$, and $\mathrm{H} 4$, were obtained. The results of the induction activity test showed that only $\mathrm{H} 4$ showed induction activity. $\mathrm{H} 4$, corresponding to the highest peak in HPLC, was evaluated by analytical HPLC, which confirmed that the single peak in $\mathrm{H} 4$ was a single pure chemical (Figure A1).

$\mathrm{MS} / \mathrm{MS}$ and protein sequence analyses were used to analyze the peptide sequence. The molecular weight of the component, as determined by LC-TOF MS, was

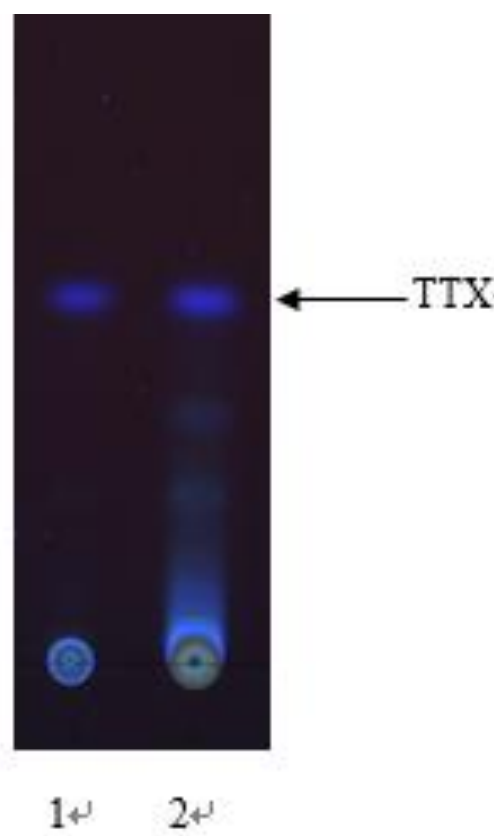

Figure 2. The TLC analysis of TTX. Lane 1: TTX standard; 2: the test sample.

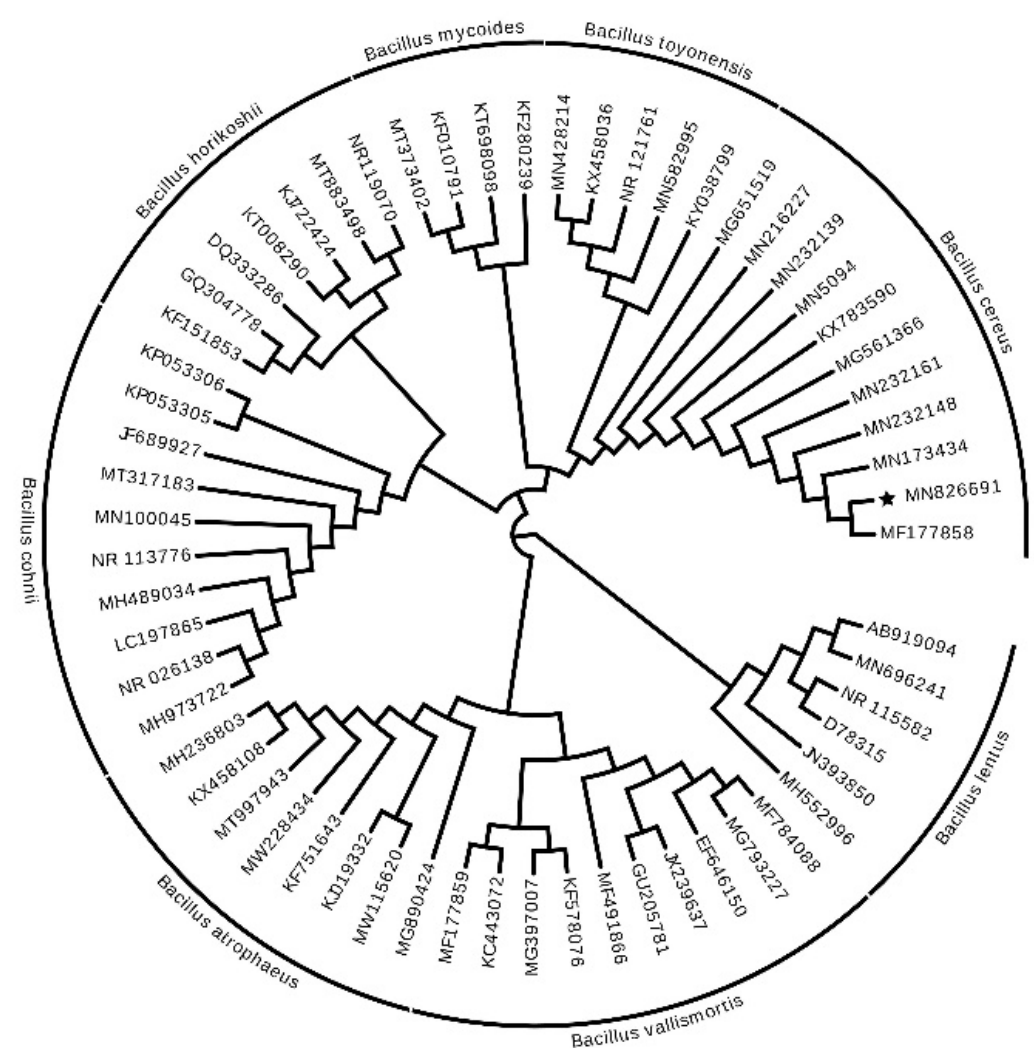

Figure 3. The annular phylogenetic tree of the strain He-1. 16S rDNA phylogenetic analysis of the strain He-1, Bacillus cereus, MN826691. 
1,188 (Figure A2), and amino acid sequencing showed that its primary structure was Leu-Gly-Tyr-Leu-Pro-ThrAla-Val-Pro-Gly-Val-Thr.

\section{Discussion}

A cost-effective, environmentally friendly, and stable method of producing TTX is highly needed. The theory that TTX is produced by symbiotic microorganisms living inside TTX-bearing organisms is gaining more acceptance (Maran et al., 2007). Most of the TTX-producing bacteria have been isolated from pufferfish inhabiting mainly the sea or coastal waters. Every spring, they migrate from the East sea of China to the coastal areas of South China to spawn. Both the quantity and toxicity of TTX in pufferfish are the highest during this period. However, only limited studies have investigated TTX-producing bacteria in the local pufferfish populations of Zhejiang, China.

Most TTX-producing bacteria belong to the genera Vibrio, Pseudomonas, Alteromonas, Flavobacterium, and Micrococcus. However, in a recent study, Lu \& Yi isolated a strain of Bacillus horikoshii from the liver of a pufferfish that produced TTX (Lu \& Yi, 2009). Moreover, Wang \& Fan described the characterization of a Bacillus strain capable of producing TTX that was isolated from the pufferfish Fugu obscurus (Wang et al., 2010). These findings, along with our current results, suggest that Bacillus species might be parasitic or symbiotic bacteria of pufferfish that produce TTX during growth.

The cultivation conditions for bacteria in the laboratory are vastly different from those in their natural habitats. We hope that through further studies, high-yielding TTX-producing strains will be isolated and fermentation conditions will be optimized to better mimic the natural environment of TTX-producing strains.

Although some achievements have been made in research on the production of TTX through microbial fermentation, there are still two main problems: (1) The toxicity of the strain (i.e., toxin production) is not stable. Previous studies have reported that although wild symbiotic strains can produce high levels of TTX, these levels markedly decrease after isolation and purification. Therefore, wild strains in early generations have strong TTX production capacity, but after several subcultures, this capacity is reduced or even completely lost, leading to difficulties in further research and applications. (2) The yield of TTX is still extremely low (nanograms). Although mutagenesis-based breeding of strains and optimization of fermentation conditions have increased yield, the level obtained from a pure culture of a single strain is still far from that needed for practical application. It is difficult to solve these two

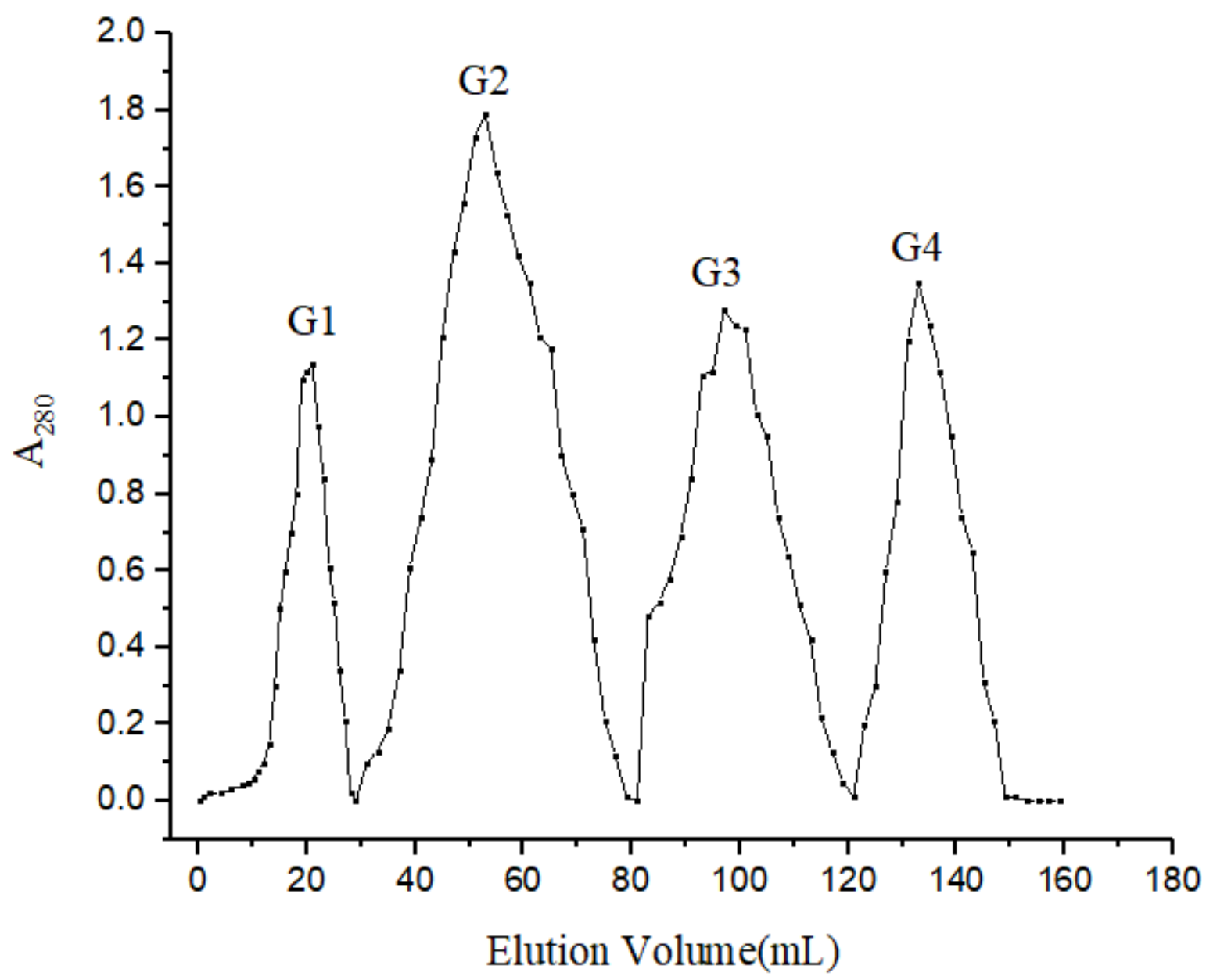

Figure 4. The gel chromatography of sample U2. 
problems through mutagenesis-based breeding and optimization of fermentation parameters. To address these issues, we must begin to better understand the nature of TTX production via microbial metabolism. Thus, to achieve new breakthroughs, the biosynthetic pathway of TTX and the related regulatory mechanisms should be studied.

There are many methods for the detection and identification of TTX. Quantitative detection methods include mouse biological assay, ELISA kit-based detection, and HPLC-fluorescence spectrophotometry. Qualitative methods include TLC and HPLC-UV spectrophotometry. Usually, a TTX standard is required for qualitative methods. In our study, a combination of different methods was used. The mouse biological assay was used for screening, which can rapidly and efficiently determine the presence of TTX in samples of microbial culture medium, and the ELISA kit was used for quantitative detection of TTX in culture medium after the preliminary purification, as it provides a more accurate measurement of TTX content. To determine whether TTX was present in the final sample, TLC was used to compare it with the TTX standard, which is easy to perform and provides an accurate result.

Traditional microbial identification methods, such as staining, culture, and simple biochemical tests, rely on phenotypic identification. Although these traditional identification methods are still widely used, they have two main disadvantages. They can be applied only to organisms that can be grown in vitro, and some strains have unique biochemical characteristics that do not fit the known species pattern. In contrast, modern molecular methods do not rely on living cultures, and they can often reveal subtle differences between organisms that cannot be detected by the traditional methods. At present, real-time fluorescence quantitative PCR is probably the most widely used molecular technique for microbial identification. PCR technology can be used to quickly detect and identify microbial species, thus speeding up diagnostic procedures. Most PCR-based methods utilize a set of universal PCR primers to identify microbial samples by sequencing a PCR-amplified sequence. 16S rRNA gene sequencing is the gold standard method for PCR-based identification of bacteria, and the internal transcriptional spacer (ITS) region is the main barcode marker for fungal species. In the present study, both traditional detection and a modern PCR method were used to identify the TTX-producing strain, which provided an accurate identification.

An inducer is an effector that stimulates activation of an operon. In microbial anabolism, the inducer may be a substrate or an intermediate in the synthetic pathway of the target metabolite. The inducer may also be an activator or inhibitor of a key enzyme in the biosynthetic pathway. Numerous studies have shown that many microorganisms require an inducer to yield a target product. At present, the microbial biosynthetic pathway of TTX is not completely understood, and the enzymes that are involved in TTX synthesis are unknown. Therefore, determining the substances that induce TTX production would be conducive to understand the TTX biosynthetic pathway from a new perspective. Our preliminary study detected an inducer in the medium from the TTX-producing strain $\mathrm{He}-1$ that could restore TTX production capacity in the non-TTXproducing derivative strain He-2. However, further analyses are needed to determine the specific mechanism.

In Edman reaction, the $\mathrm{N}$-terminal amino acid residues of a peptide are degraded and then extracted with organic solvents; thus, each amino acid can be analyzed. Specifically, the peptide to be sequenced is adsorbed on a solid surface, and phenyl isothiocyanate is added to the adsorbed peptide with a mild alkaline buffer solution of $12 \%$ trimethylamine to react with the amine group of the $\mathrm{N}$-terminal amino acid. The terminal amino acid is then selectively separated by the addition of anhydrous acid. The derivatives are then isomerized to yield substituted phenylacetonylurea, which can be eluted and identified by chromatography, following which the cycle can be repeated. Each step has an efficiency of approximately $98 \%$, enabling the reliable determination of about 50 amino acids. In this study, the sequence was determined directly with a protein sequence analyzer. The peptide sample was immobilized in the reaction vessel of the protein sequencer and subjected to Edman degradation. Each cycle released an amino acid from the $\mathrm{N}$-terminus of the peptide, and the released amino acid derivatives were identified by HPLC. This process was repeated for the entire peptide until the entire measurable sequence or predetermined number of cycles was completed.

\section{Ethical Statement}

The guidelines established by the Administration of Affairs Concerning Animal Experimentation state that approval from the Science and Technology Bureau of China and the Department of Wildlife Administration is not necessary when the fish in question are neither rare nor near extinction (first-or second-class state protection level). Therefore, approvalwas not required for the experiments conducted in this study.

\section{Funding Information}

Work supported by the Natural Science Foundation of Zhejiang Province under Grant number LY17C010001; the Natural Science Foundation of Ningbo under Grant number 2017A610287.

\section{Author Contribution}

Yong-jun Yuan, Chen Yu, and Zhuo Chen conducted the experiments; Feng Guan analyzed the data and wrote the manuscript; Jie Zhang contributed to data 
analysis and revising the manuscript; Yong-jun Yuan supervised the whole project.

\section{Conflict of Interest}

The authors declare that they have no known competing financial or non-financial, professional, or personal conflicts that could have appeared to influence the work reported in this paper.

\section{References}

Bane, V., Lehane, M., Dikshit, M., O'Riordan, A., \& Furey, A. (2014). Tetrodotoxin: chemistry, toxicity, source, distribution and detection. Toxins, 6(2), 693-755. https://doi.org/10.3390/toxins6020693

Campbell, S., Harada, R. M., DeFelice, S. V., Bienfang, P. K., \& Li, Q. X. (2009). Bacterial production of tetrodotoxin in the pufferfish Arothron hispidus. Natural Product Research, 23(17), 1630-1640. https://doi.org/10.1080/14786410903003780

Chau, R., Kalaitzis, J. A., \& Neilan, B. A. (2011). On the origins and biosynthesis of tetrodotoxin. Aquatic Toxicology, 104(1-2), 61-72. https://doi.org/10.1016/j.aquatox.2011.04.001

Hagen, N. A., du Souich, P., Lapointe, B., Ong-Lam, M., Dubuc, B., Walde, D., Love, R., \& Ngoc, A. H. (2008). Tetrodotoxin for moderate to severe cancer pain: a randomized, double blind, parallel design multicenter study. Journal of Pain and Symptom Management, 35(4), 420-429. https://doi.org/10.1016/j.jpainsymman.2007.05.011

Hwang, D. F., \& Noguchi, T. (2007). Tetrodotoxin poisoning. Advances in Food and Nutrition Research, 52, 141-236. https://doi.org/10.1016/S1043-4526(06)52004-2

Kudo, Y., Finn, J., Fukushima, K., Sakugawa, S., Cho, Y., Konoki, K., \& Yotsu-Yamashita, M. (2014). Isolation of 6deoxytetrodotoxin from the pufferfish, Takifugu pardalis, and a comparison of the effects of the C- 6 and C-11 hydroxy groups of tetrodotoxin on its activity. Journal of Ntural Poducts, 77(4), 1000-1004. https://doi.org/10.1021/np401097n

Lu, Y., \& Yi, R. (2009). Bacillus horikoshii, a tetrodotoxinproducing bacterium isolated from the liver of puffer fish. Annals of Mcrobiology, 59(3), 453-458. https://doi.org/10.1007/BF03175130

Magarlamov, T. Y., Beleneva, I. A., Chernyshev, A. V., \& Kuhlevsky, A. D. (2014). Tetrodotoxin-producing Bacillus $\mathrm{sp}$. from the ribbon worm (Nemertea) Cephalothrix simula (Iwata, 1952). Toxicon, 85, 46-51. https://doi.org/10.1016/j.toxicon.2014.04.015

Maran, B. A. V., Iwamoto, E., Okuda, J., Matsuda, S., Taniyama, S., Shida, Y., Asakawa, M., Ohtsuka, S., Nakai, T., \& Boxshall, G. A. (2007). Isolation and characterization of bacteria from the copepod Pseudocaligus fugu ectoparasitic on the panther puffer Takifugu pardalis with the emphasis on TTX. Toxicon, 50(6), 779-790. https://doi.org/10.1016/j.toxicon.2007.06.021

Nieto, F. R., Cobos, E. J., Tejada, M. Á., Sánchez-Fernández, C., González-Cano, R., \& Cendán, C. M. (2012). Tetrodotoxin
(TTX) as a therapeutic agent for pain. Marine Dugs, 10(2), 281-305. https://doi.org/10.3390/md10020281

Nishikawa, T., \& Isobe, M. (2013). Synthesis of tetrodotoxin, a classic but still fascinating natural product. The Chemical Record, 13(3), 286-302. https://doi.org/10.1002/tcr.201200025

Noguch, T., \& Arakawa, O. (2008). Tetrodotoxin-distribution and accumulation in aquatic organisms, and cases of human intoxication. Marine Dugs, 6(2), 220-242. https://doi.org/10.3390/md6020220

Padera, R. F., Tse, J. Y., Bellas, E., \& Kohane, D. S. (2006). Tetrodotoxin for prolonged local anesthesia with minimal myotoxicity. Muscle \& Nerve, 34(6), 747-753. https://doi.org/10.1002/mus.20618

Pratheepa, V., \& Vasconcelos, V. (2013). Microbial diversity associated with tetrodotoxin production in marine organisms. Environmental Txicology and Parmacology, 36(3), 1046-1054. https://doi.org/10.1016/j.etap.2013.08.013

Rocher, A., Caceres, A. I., Obeso, A., \& Gonzalez, C. (2011). Tetrodotoxin as a tool to elucidate sensory transduction mechanisms: the case for the arterial chemoreceptors of the carotid body. Marine Dugs, 9(12), 2683-2704. https://doi.org/10.3390/md9122683

Sanford, L. D., Yang, L., Tang, X., Ross, R. J., \& Morrison, A. R. (2005). Tetrodotoxin inactivation of pontine regions: Influence on sleep-wake states. Brain Rsearch, 1044(1), 42-50. https://doi.org/10.1016/j.brainres.2005.02.079

Song, H., Li, J., Lu, C. L., Kang, L., Xie, L., Zhang, Y. Y., Zhou, X. B., \& Zhong, S. (2011). Tetrodotoxin alleviates acute heroin withdrawal syndrome: A multicentre, randomized, double-blind, placebo-controlled study. Clinical and Experimental Pharmacology and Physiology, 38(8), 510-514. https://doi.org/10.1111/j.14401681.2011.05539.x

Steffens, H., Eek, B., Trudrung, P., \& Mense, S. (2003). Tetrodotoxin block of A-fibre afferents from skin and muscle-a tool to study pure C-fibre effects in the spinal cord. Pflügers Archiv, 445(5), 607-613. https://doi.org/10.1007/s00424-002-0970-2

Tu, N., Tu, Q., Tung, H., Hieu, D., \& Romero-Jovel, S. (2014). Detection of tetrodotoxin-producing Providencia rettgeri T892 in Lagocephalus pufferfish. World Journal of Microbiology and Biotechnology, 30(6), 1829-1835. https://doi.org/10.1007/s11274-014-1601-8

Turner, A. D., Powell, A., Schofield, A., Lees, D. N., \& BakerAustin, C. (2015). Detection of the pufferfish toxin tetrodotoxin in European bivalves, England, 2013 to 2014. Eurosurveillance, 20(2), 21009. https://doi.org/10.2807/1560-7917.ES2015.20.2.21009

Wang, J., Fan, Y., \& Yao, Z. (2010). Isolation of a Lysinibacillus fusiformis strain with tetrodotoxin-producing ability from puffer fish Fugu obscurus and the characterization of this strain. Toxicon, 56(4), 640-643. https://doi.org/10.1016/j.toxicon.2010.05.011

Yu, V. C. H., Yu, P. H. F., Ho, K. C., \& Lee, F. W. F. (2011). Isolation and identification of a new tetrodotoxinproducing bacterial species, Raoultella terrigena, from Hong Kong marine puffer fish Takifugu niphobles. Marine Drugs, 9(11), 2384-2396. https://doi.org/10.3390/md9112384 